\title{
Generalized qudit Choi maps
}

\author{
M. A. Jafarizadeh ${ }^{a, b, c} *$ M. Rezaee ${ }^{a, c} \dagger$ and S. Ahadpour ${ }^{a, c \ddagger}$. \\ ${ }^{a}$ Department of Theoretical Physics and Astrophysics, Tabriz University, Tabriz 51664, Iran. \\ ${ }^{b}$ Institute for Studies in Theoretical Physics and Mathematics, Tehran 19395-1795, Iran. \\ ${ }^{c}$ Research Institute for Fundamental Sciences, Tabriz 51664, Iran.
}

October 9, 2018

*E-mail:jafarizadeh@tabrizu.ac.ir
${ }^{\dagger}$ E-mail:karamaty@tabrizu.ac.ir
${ }^{\ddagger}$ E-mail:s.ahadpour@tabrizu.ac.ir 


\begin{abstract}
Following the linear programming prescription of Ref. [6], the $d \otimes d$ Bell diagonal entanglement witnesses are provided. By using Jamiolkowski isomorphism, it is shown that the corresponding positive maps are the generalized qudit Choi maps. Also by manipulating particular $d \otimes d$ Bell diagonal separable states and constructing corresponding bound entangled states, it is shown that thus obtained $d \otimes d$ BDEW's (consequently qudit Choi maps) are non-decomposable in certain range of their parameters. Keywords: Entanglement witness, Bell state, Generalized Choi map. Non decomposable PACs Index: 03.65.Ud
\end{abstract}




\section{Introduction}

Entanglement is one of the most fascinating features of quantum mechanics. As Einstein, Podolsky and Rosen [1] pointed out, the quantum states of two physically separated systems that interacted in the past can defy our intuitions about the outcome of local measurements. Moreover, it has recently been recognized that entanglement is a very important resource in quantum information processing[2]. A bipartite mixed state is said to be separable [3] (not entangled) if considered as a convex combination of pure product states.

One of the approaches to distinguish separable states from entangled ones involves the so called entanglement witness (EW) [4]. An EW for a given entangled state $\rho$ is an observable W expectation value of which is nonnegative on any separable state, but strictly negative on an entangled state $\rho$.

There is a correspondence relating entanglement witnesses to linear positive (but not completely positive) maps from the operators on Hilbert space $H_{A}$ to the operators on Hilbert space $H_{B}$ via Jamiolkowski isomorphism, or vice versa[5].

Here in this paper, by using the prescription of Ref[6], i.e., reducing the manipulation of generic Bell-state diagonal-entanglement witnesses to an optimization problem, we find $d \otimes d$ Bell states diagonal entanglement witnesses (BDEW). By using Jamiolkowski isomorphism, we show that corresponding positive map is the generalized qudit Choi map. Also by manipulating particular qudit Bell diagonal separable states and constructing corresponding bound entangled states, we show that thus obtained $d \otimes d$, BDEW's (consequently qudit Choi maps) are nondecomposable in certain range of their parameters.

The paper is organized as follows:

In section 2, we introduce the the $d \otimes d$ Bell diagonal separable states and corresponding PPT states. In section 3 we give a brief review of entanglement witness and we show that finding generic Bell states diagonal entanglement witnesses for $d \otimes d$ systems reduces to a LP problem. 
In section 4 and 5 we find generalized $d \otimes d$ Choi entanglement witnesses of first and second types, respectively. Section 6, is devoted to investigation of non-decomposability of generalized Choi entanglement witnesss. The paper is ended with a brief conclusion.

\section{$2 \quad$ Bell diagonal $d \otimes d$ separable states}

Here in this section we introduce some sets of the $d \otimes d$ Bell diagonal separable states(BDSS). In general the set of BDSS consists of the following three categories:

Set 1: The first set of BDSS can be constructed from Bell states

$$
\left|\psi_{k 0}\right\rangle=\frac{1}{\sqrt{d}} \sum_{l=0}^{d-1}\left(\omega^{k}\right)^{l}|l\rangle|l\rangle
$$

where $\omega=e^{\frac{2 \pi i}{d}}$ and $k=0,1, \ldots, d-1$, simply by summing over the following Bell states projection operators:

$$
\sum_{k=0}^{d-1}\left|\psi_{k 0}\right\rangle\left\langle\psi_{k 0}\left|=\frac{1}{d} \sum_{l, l^{\prime}=0}^{d-1} d \delta_{l, l^{\prime}}\right| l\right\rangle|l\rangle \otimes\left\langle l^{\prime}\right|\left\langle l^{\prime}\right|,
$$

where we obtain one of the BDSS of first type, defined as

$$
\rho_{0}=\sum_{k=0}^{d-1}\left|\psi_{k 0}\right\rangle\left\langle\psi_{k 0}\left|=\sum_{l=0}^{d-1}\right| l\right\rangle\langle l|\otimes| l\rangle\langle l| .
$$

Acting Shift operator on (2-3) we can find the other BDSS of first type as follows

$$
\rho_{m}=\left(I_{d} \otimes S^{m}\right) \sum_{k=0}^{d-1}\left|\psi_{k 0}\right\rangle\left\langle\psi_{k 0}\right|\left(I_{d} \otimes S^{m}\right)^{\dagger}, \quad m=1,2, \ldots, d-1 .
$$

Therefore, the first set of BDSS consists of the following d BDSS

$$
\rho_{m}=\sum_{k=0}^{d-1}\left|\psi_{k m}\right\rangle\left\langle\psi_{k m}\left|=\sum_{l=0}^{d-1}\right| l\right\rangle\langle l|\otimes| l+m\rangle\langle l+m|, \quad m=0,1, \ldots, d-1 .
$$

\section{Set 2:}

In order to obtain the second set of BDSS, we need to consider the following sum of projection operators

$$
\phi_{k}^{\prime}=\sum_{i=0}^{d-1}\left|v_{i}\right\rangle \sum_{j=0}^{d-1}\left|v_{j}\right\rangle \otimes \sum_{l=0}^{d-1}\left\langle v_{l}\right| \sum_{m=0}^{d-1}\left\langle v_{m}\right|
$$


where $\left|v_{i}\right\rangle=\frac{\left(\omega^{k}\right)^{i}|i\rangle}{\sqrt{d}}$.

Now summing over the free index $\mathrm{k}$ and doing some routine calculations we obtain the following BDSS of second type

$$
\rho_{0}^{\prime}=\sum_{k=0}^{d-1} \phi_{k}^{\prime}=\sum_{k=0}^{d-1}\left(\sum_{i=0}^{d-1} \frac{1}{\sqrt{d}}|i\rangle|i+k\rangle\right) \otimes\left(\sum_{j=0}^{d-1} \frac{1}{\sqrt{d}}\langle j|\langle j+k|\right),
$$

where it can be written in terms of Bell states as

$$
\rho_{0}^{\prime}=\sum_{k=0}^{d-1}\left|\psi_{0 k}\right\rangle\left\langle\psi_{0 k}\right|
$$

The remaining BDSS of second type can be obtained by applying powers of Modulation operator over (2-7) separable state,

$$
\rho_{m}^{\prime}=\sum_{k=0}^{d-1}\left(\Omega^{m} \otimes I_{d}\right)\left|\psi_{0 k}\right\rangle\left\langle\psi_{0 k}\right|\left(\Omega^{m} \otimes I_{d}\right)^{\dagger}, \quad m=1,2, \ldots, d-1 .
$$

Hence, the second set of BDSS consists of the following d BDSS

$$
\rho_{m}^{\prime}=\sum_{k=0}^{d-1}\left|\psi_{m k}\right\rangle\left\langle\psi_{m k}\left|=\sum_{i, j, k=0}^{d-1} \omega^{m(i-j)}\right| i\right\rangle|i+k\rangle \otimes\langle j|\langle j+k|, \quad m=0,1, \ldots, d-1 .
$$

Set 3: Finally in order to obtain the third set of BDSS, we first define the following set of vectors

$$
\begin{aligned}
& \left|u_{i}\right\rangle=a_{i}\left(\omega^{k}\right)^{i}|i\rangle, \\
& \left|w_{i}\right\rangle=b_{i}\left(\omega^{k}\right)^{i}|i\rangle,
\end{aligned}
$$

then using above vectors, we construct the following sum of projection operators

$$
\phi_{k}^{\prime \prime}=\left(\sum_{i=0}^{d-1} a_{i}\left(\omega^{k}\right)^{i}|i\rangle\right)\left(\sum_{j=0}^{d-1} \overline{a_{j}}\left(\omega^{-k}\right)^{j}\langle j|\right) \otimes\left(\sum_{i=0}^{d-1} b_{l}\left(\omega^{k}\right)^{l}|l\rangle\right)\left(\sum_{m=0}^{d-1} \overline{b_{m}}\left(\omega^{-k}\right)^{m}\langle m|\right),
$$

where we assume that $a_{i}=\bar{b}_{i}$ and $a_{i+1}=\omega^{-n i} a_{i}$. Now, summing over free index $\mathrm{k}$ and using the relation $\left|a_{i}\right|^{2}=\frac{1}{\sqrt{d}}$ and doing some straightforward calculations we obtain the following BDSS's of third type

$$
\left.\rho_{n}^{\prime \prime}=\sum_{i, j, k=0}^{d-1} \omega^{n k i} \frac{1}{\sqrt{d}}|i\rangle|i+k\rangle \otimes \frac{1}{\sqrt{d}}\langle j|\langle j+k|\right) \omega^{-n k j},
$$


where it can be written in terms of Bell states as

$$
\rho_{n}^{\prime \prime}=\sum_{k=0}^{d-1}\left(\omega^{n}\right)^{\frac{k(k+1)}{2}}\left|\psi_{n k, k}\right\rangle\left\langle\psi_{n k, k}\right|\left(\omega^{-n}\right)^{\frac{k(k+1)}{2}} .
$$

Therefore, the third set of separable states are defined as

$$
\rho_{n}^{\prime \prime}=\sum_{k=0}^{d-1}\left|\psi_{n k, k}\right\rangle\left\langle\psi_{n k, k}\right|,
$$

where $\rho_{0}^{\prime \prime}=\rho_{0}^{\prime}$. So far we have introduced (3d-1) separable states. Other separable states can be obtained by applying powers of local Shift $(S)$ or Modulation $(\Omega)$ operators on these separable states. For instance we consider $3 \otimes 3$ system which consists of 8 separable states. Now, we can obtain some other separable states simply by acting powers of Shift operator on above defined separable states as

$$
\begin{gathered}
\left.\left(I_{3} \otimes S^{2}\right)\right) \rho_{1}^{\prime \prime}\left(I_{3} \otimes S^{2}\right)^{\dagger}=|10\rangle\langle 10|+| 02\rangle\langle 02|+| 21\rangle\langle 21|, \\
\left.\left(I_{3} \otimes S^{2}\right)\right) \rho_{2}^{\prime \prime}\left(I_{3} \otimes S^{2}\right)^{\dagger}=|20\rangle\langle 20|+| 02\rangle\langle 02|+| 11\rangle\langle 11|, \\
\left.\left(I_{3} \otimes S\right)\right) \rho_{1}^{\prime \prime}\left(I_{3} \otimes S\right)^{\dagger}=|01\rangle\langle 01|+| 12\rangle\langle 12|+| 20\rangle\langle 20|, \\
\left.\left(I_{3} \otimes S\right)\right) \rho_{2}^{\prime \prime}\left(I_{3} \otimes S\right)^{\dagger}=|01\rangle\langle 01|+| 22\rangle\langle 22|+| 10\rangle\langle 10| .
\end{gathered}
$$

It is easy to see that some of thus obtained BDSS's lie at the boundary of separable states [6]. One can show that the $\rho_{S}^{1}=\rho_{0}, \rho_{S}^{2}=\rho_{0}^{\prime}=\rho_{0}^{\prime \prime}$ are orthogonal to the optimal $W_{\text {red }}$; i.e., we have $\operatorname{Tr}\left[W_{\text {red }} \rho_{S}^{i}\right]=0, i=1,2$. Hence, $\rho_{S}^{i}, i=1,2$ lie at the boundary of the separable region. Also convex sum of these states i.e, $\rho_{\lambda}=\lambda \rho_{S}^{1}+(1-\lambda) \rho_{S}^{2}$ is orthogonal to the optimal $W_{\text {red }}$; i.e., we have $\operatorname{Tr}\left[W_{\text {red }} \rho_{\lambda}\right]=0$. Hence, $\rho_{\lambda}$ lies at the boundary of the separable region.

At the end of this section we try to introduce some positive partial transpose (PPT) operators states which will be used later, in the investigation of non-decomposability of generalized Choi entanglement witnesss. We consider the following $d \otimes d$ density matrix defined as

$$
\rho_{P P T}=p\left(\left|\psi_{00}\right\rangle\left\langle\psi_{00}\right|\right)+\frac{1-p}{d}\left(\mu_{1} \rho_{1}+\mu_{2} \rho_{2}+\ldots+\mu_{d-1} \rho_{d-1}\right)
$$

where it is positive for $0 \leq p, \mu_{1}, \mu_{2}, ., ., \mu_{d-1} \leq 1$ and $\sum_{i=1}^{d-1} \mu_{i}=1$. Now in order to make the partial transpose of density matrix (2-18) to be positive, i.e., to obtain PPT density matrix, we 
need to use the relations $\rho_{i}^{T_{A}}=\rho_{i}$ and substitute the partial transpose of Bell state projection operator $\left(\left|\psi_{00}\right\rangle\left\langle\psi_{00}\right|\right)$

$$
\left(\left|\psi_{00}\right\rangle\left\langle\psi_{00}\right|\right)^{T_{A}}=\frac{1}{d} \sum_{m, l=0}^{d-1} \omega^{m l}\left|\psi_{m, l}\right\rangle\left\langle\psi_{m, d-(l)}\right|,
$$

in partial transpose of Eq.(2-18). Now, the positivity of partial transpose of density matrix (2-18) implies that

$$
\rho_{P P T}^{T_{A}} \geq 0 \Rightarrow p \leq \max \left\{\min \left\{\frac{\mu_{i}}{1+\mu_{i}}, \frac{\sqrt{\mu_{k} \mu_{j}}}{1+\sqrt{\mu_{k} \mu_{j}}}\right\}\right\} \quad, \quad k \neq i \neq j=1, \ldots, d-1 .
$$

For $\mu_{1}=\mu_{2}=\ldots=\mu_{d-1}=\frac{1}{d-1}$ the parameter $\mathrm{p}$ is optimal and equal to $p=\frac{1}{d}$, where $d \otimes d$ density matrix reduces to

$$
\rho_{P P T}=\left[p\left(\left|\psi_{00}\right\rangle\left\langle\psi_{00}\right|\right)+\frac{1-p}{d} \sum_{i=1}^{d-1} \mu_{i} \rho_{i}\right], p \leq \frac{1}{d} .
$$

As we will show later in section 6 , for certain range of parameter $\mathrm{p}$ the density matrix (2-18) become entangled (actually bound entangled due to its PPT property).

In remaining part of this section we consider particular case of $d=3$

$$
\rho_{P P T}=p\left(\left|\psi_{00}\right\rangle\left\langle\psi_{00}\right|\right)+\frac{1-p}{3}\left(\mu_{1} \rho_{1}+\mu_{2} \rho_{2}\right)
$$

where the positivity of its partial transpose implies that

$$
p \leq \max \left\{\frac{\sqrt{\mu_{2} \mu_{1}}}{1+\sqrt{\mu_{2} \mu_{1}}}\right\},
$$

where for $\mu_{1}=\mu_{2}=\frac{1}{2}$ the parameter $\mathrm{p}$ is optimal and it is equal to $p=\frac{1}{3}$.

Finally we construct $3 \otimes 3$ PPT state of second type by using (2-10) separable states as

$$
\rho_{P P T}^{\prime}=p\left(\left|\psi_{00}\right\rangle\left\langle\psi_{00}\right|\right)+\frac{1-p}{3}\left(\mu_{1} \rho_{1}^{\prime}+\mu_{2} \rho_{2}^{\prime}\right)
$$

where it is positive for $0 \leq p, \mu_{1}, \mu_{2} \leq 1$ and $\sum_{i=1}^{2} \mu_{i}=1$.

Now, the positivity of partial transpose of density matrix (2-24) implies that

$$
\rho_{P P T}^{T_{A}} \geq 0 \Rightarrow p \leq \max \left\{\frac{2 \mu_{1}^{2}-2+2 \mu_{2}^{2}-2 \mu_{1} \mu_{2}+3 \mu_{2}+3 \mu_{1}+3 \sqrt{4+6 \mu_{1} \mu_{2}-3 \mu_{1}^{2}-3 \mu_{2}^{2}}}{2\left(8+\mu_{2}^{2}-\mu_{1} \mu_{2}+\mu_{1}^{2}+3 \mu_{1}+3 \mu_{2}\right)}\right\} .
$$

For $\mu_{1}=\mu_{2}=\frac{1}{2}$ the parameter $\mathrm{p}$ is maximum and equal to $p=\frac{1}{3}$. 


\section{$3 \quad$ Bell state diagonal $d \otimes d$ entanglement witness}

In this section we give a brief outline of some of the main features of Bell state diagonal $d \otimes d$ entanglement witness together with linear programming prescription for finding it. For further information reader is referred to Ref.[6].

Let $\mathrm{S}$ be a convex compact set in a finite dimensional Banach space. Let $\rho$ be a point in the space with $\rho$ which is not in $S$. Then there exists a hyperplane that separates $\rho$ from $\mathrm{S}$ $[5,7,8]$.

A hermitian operator (an observable) W is called an entanglement witness (EW) iff

$$
\begin{gathered}
\exists \rho \text { such that } \operatorname{Tr}(\hat{\rho} W)<0 \\
\forall \rho^{\prime} \in S \quad \operatorname{Tr}\left(\rho^{\prime} \hat{W}\right) \geq 0 .
\end{gathered}
$$

Using these definitions, we can restate the consequences of the Hahn-Banach theorem [8] in several ways:

1- $\rho$ is entangled iff there exists a witness $\mathrm{W}$ such that $\operatorname{Tr}(\rho W)<0$.

2- $\rho$ is a PPT entangled state iff there exists a non-decomposable entanglement witness W such that $\operatorname{Tr}(\rho W)<0$.

3- $\sigma$ is separable iff for all EW $\operatorname{Tr}(W \sigma) \geq 0$.

From theoretical point of view this theorem is quite powerful. However, it is not useful to construct witnesses that detect a given state $\rho$.

We know that a strong relation was developed between entanglement witnesses and positive maps $[5,7]$. Notice that an entanglement witness only gives one condition (namely $\operatorname{Tr}(W \rho)<0)$ while for the map $\left(I_{A} \otimes \phi\right) \rho$ to be positive definite, there are many conditions that have to be satisfied. Thus the map is much stronger, while the witnesses are much weaker in detecting entanglement. It is shown that this concept is able to provide a more detailed classification of entangled states. 
Following Ref[6], one can expand any trace class observable in the Bell basis as

$$
W=\sum_{i_{1} i_{2}=0}^{d-1} W_{i_{1} i_{2}}\left|\psi_{i_{1} i_{2}}\right\rangle\left\langle\psi_{i_{1} i_{2}}\right|
$$

where $\left|\psi_{i_{1} i_{2}}\right\rangle$ for $\left(0 \leq i_{1} \leq d, 0 \leq i_{2} \leq d\right)$ stands for the orthonormal states for a $d \otimes d$ Bell state. After some calculations similar to those of [6], the trace-1 Bell state diagonal W observable can be written as

$$
W=\mathbf{r} \frac{I_{d^{2}}}{d^{2}}+(1-\mathbf{r}) \sum_{i_{1} i_{2}=0}^{d-1} q_{i_{1} i_{2}}\left|\psi_{i_{1} i_{2}}\right\rangle\left\langle\psi_{i_{1} i_{2}}\right| .
$$

The observable given by (3-29) is not a positive operator and can not be an EW provided that its expectation value on any pure product state is positive. For a given product state $|\gamma\rangle=|\alpha\rangle_{1}|\alpha\rangle_{2}$ the non negativity of

$$
\operatorname{Tr}(W|\gamma\rangle\langle\gamma|) \geq 0
$$

implies that

$$
\frac{-d^{2} \sum_{i_{1} i_{2}=0}^{d-1} q_{i_{1} i_{2}} P_{i_{1} i_{2}}}{1-d^{2} \sum_{i_{1} i_{2}=0}^{d-1} q_{i_{1} i_{2}} P_{i_{1} i_{2}}} \leq \mathbf{r} \leq 0
$$

where $P_{i_{1} i_{2}}=|<\gamma| \psi_{i_{1} i_{2}}>\left.\right|^{2}$.

As for the completeness of the Bell state $\sum_{i_{1} i_{2}}\left|\psi_{i_{1} i_{2}}\right\rangle\left\langle\psi_{i_{1} i_{2}}\right|=1$, the determination of $\mathbf{r}_{c}$ reduces to the following optimization problem[10]

$$
\begin{gathered}
\operatorname{minimize} \quad C_{\gamma}=\sum_{i_{1} i_{2}} q_{i_{1} i_{2}} P_{i_{1} i_{2}}(\gamma) \\
0 \leq P_{i_{1} i_{2}}(\gamma) \leq \frac{1}{d} \\
\sum_{i_{1} i_{2}} P_{i_{1} i_{2}}(\gamma)=1
\end{gathered}
$$

Always the distribution $P_{i_{1} i_{2}}$ satisfies $0 \leq P_{i_{1} i_{2}}(\gamma) \leq \frac{1}{d}$ for all pure product states[6]. One can calculate the distributions $P_{i_{1} i_{2}}(\gamma)$, consistent with the aforementioned optimization problem, from the information about the boundary of feasible region. To achieve the feasible region we obtain the extreme points corresponding to the product distributions $P_{i_{1} i_{2}}(\gamma)$ for every given product states by applying the special conditions on $q_{i_{1} i_{2}}$ 's parameters. $C_{\gamma}$ themselves are 
functions of the product distributions, and they are in turn are functions of $\gamma$. They are not real variables of $\gamma$ but the product states will be multiplicative. If this feasible region constructs a polygon by itself, the corresponding boundary points of the convex hull will minimize exactly $C_{\gamma}$ in Eq. (3-32). This problem is called LP , and the simplex method is the easiest way of solving it. If the feasible region is not a polygon, with the help of tangent planes in this region at points which are determined either analytically or numerically one can define new convex hull which is a polygon and has encircled the feasible region. The points on the boundary of the polygon can approximately determine the minimum value $C_{\gamma}$ from Eq.(3-32). Thus approximated value is obtained via LP.

\section{Generalized qudit Choi map of first type}

Compared with qutrit Choi positive map [9] $\phi(a, b, c): M^{3} \rightarrow M^{3}$, Generalized qudit Choi map of first type $\phi\left(a_{0}, \cdots, a_{d-1}\right): M^{d} \rightarrow M^{d}$ is defined as

$$
\begin{gathered}
\phi_{a_{0}, \cdots, a_{d-1}}(\rho)= \\
\left(\begin{array}{cccc}
a_{0} \rho_{11}+a_{1} \rho_{22}+\cdots+a_{d-1} \rho_{d d} & 0 & \ldots & 0 \\
0 & a_{d-1} \rho_{11}+a_{0} \rho_{22}+\cdots+a_{d-2} \rho_{d d} & \ldots & 0 \\
\vdots & \vdots & \ddots & \vdots \\
0 & 0 & \ldots & a_{1} \rho_{11}+a_{2} \rho_{22}+\cdots+a_{0} \rho_{d d}
\end{array}\right)
\end{gathered}
$$

where $\rho \in M^{d}$. Using Jamiolkowski [5] isomorphism between the positive map and the operators, we obtain the following $d \otimes d$ entanglement witnesses corresponding to Choi map

$$
\begin{gathered}
W_{\text {Choi }}= \\
\frac{1}{d\left(a_{0}+\cdots+a_{d-1}-1\right)}\left(a_{0} \sum_{k=0}^{d-1}\left|\psi_{k 0}\right\rangle\left\langle\psi_{k 0}\left|+a_{1} \sum_{k=0}^{d-1}\right| \psi_{k 1}\right\rangle\left\langle\psi_{k 1}\left|+\cdots+a_{d-1} \sum_{k=0}^{d-1}\right| \psi_{k, d-1}\right\rangle\left\langle\psi_{k, d-1}|-d| \psi_{00}\right\rangle\left\langle\psi_{00}\right|\right),
\end{gathered}
$$


where it can written in terms of separable states (2-5) in the following form

$$
W_{C h o i}=\frac{1}{d\left(a_{0}+\cdots+a_{d-1}-1\right)}\left(\sum_{m=0}^{d-1} a_{m} \rho_{m}-d\left|\psi_{00}\right\rangle\left\langle\psi_{00}\right|\right) .
$$

For product state $|\gamma\rangle=|\alpha\rangle_{1}|\alpha\rangle_{2}=\frac{1}{\sqrt{d}}\left(\begin{array}{cccccc}1 & \omega & \omega^{2} & \ldots & \omega^{d-1}\end{array}\right)^{T} \otimes \frac{1}{\sqrt{d}}\left(\begin{array}{cccccc}1 & \omega & \omega^{2} & \ldots & \omega^{d-1}\end{array}\right)^{T}$ the non negativity of (4-35) implies that

$$
a_{0}+a_{1}+\ldots+a_{d-1} \geq d
$$

Similar to BDEW we expand $\left|\psi_{00}\right\rangle\left\langle\psi_{00}\right|$ using the identity operator and the other Bell diagonal states:

$$
\left|\psi_{00}\right\rangle\left\langle\psi_{00}\left|=I_{d^{2}}-\sum_{i \neq j=0}^{d-1}\right| \psi_{i j}\right\rangle\left\langle\psi_{i j}\right| .
$$

Then we reduce EW to the following form

$$
\begin{gathered}
W_{C h o i}=\frac{1}{d\left(a_{0}+\cdots+a_{d}-1\right)}\left(-\left(d-a_{0}\right) I_{d^{2}}+d \sum_{k=1}^{d-1}\left|\psi_{k 0}\right\rangle\left\langle\psi_{k 0}\right|\right. \\
\left.+\left(a_{1}+d-a_{0}\right) \sum_{k=0}^{d-1}\left|\psi_{k 1}\right\rangle\left\langle\psi_{k 1}\left|+\cdots+\left(a_{d-1}+d-a_{0}\right) \sum_{k=0}^{d-1}\right| \psi_{k, d-1}\right\rangle\left\langle\psi_{k, d-1}\right|\right) .
\end{gathered}
$$

Comparing with BDEW (3-29) we have

$$
\mathbf{r}=-\frac{d\left(d-a_{0}\right)}{\left(a_{0}+\cdots+a_{d-1}-1\right)}
$$

and the EW operator is defined as

$$
\begin{gathered}
W_{C h o i}=\mathbf{r} \frac{I_{d^{2}}}{d^{2}}+\frac{(1-\mathbf{r})}{\left(\left(d^{2}-1\right)+(1-d) a_{0}+a_{1}+\cdots+a_{d-1}\right)}\left(\sum_{k=1}^{d-1}\left|\psi_{k 0}\right\rangle\left\langle\psi_{k 0}\left|+\left(a_{1}+d-a_{0}\right) \sum_{k=0}^{d-1}\right| \psi_{k 1}\right\rangle\left\langle\psi_{k 1}\right|\right. \\
\left.+\cdots+\left(a_{d-1}+d-a_{0}\right) \sum_{k=0}^{d-1}\left|\psi_{k, d-1}\right\rangle\left\langle\psi_{k, d-1}\right|\right) .
\end{gathered}
$$

Note that if $\mathbf{r}$ is negative, as introduced in EW above, this operator will be positive, but not a completely positive map. Using (4-36) inequality, the minimum negative eigenvalue of choi EW (4-40) is given by

$$
\frac{\mathbf{r}}{d^{2}}+(1-\mathbf{r}) \frac{a_{\min }+d-a_{0}}{d\left(\left(d^{2}-1\right)+(1-d) a_{0}+a_{1}+\cdots+a_{d-1}\right)}<0 \quad, \quad a_{\min }=\min \left\{a_{0}, a_{1}, \ldots, a_{d-1}\right\},
$$


where above inequality is satisfied for $\mathbf{r} \leq 0$ and $1 \leq a_{0} \leq d$.

By using (3-30) for non-negativity of the observable $W_{c h o i}$ we find the distributions $P_{i j}$ as a function of $q_{i j}$. The minimum value of $C_{\gamma}$ is obtained from the boundary of the feasible region, i.e., we have

$\left(C_{\gamma}\right)=\frac{1}{\left(\left(d^{2}-1\right)+(1-d) a_{0}+a_{1}+\cdots+a_{d-1}\right)}\left(\mathcal{P}_{1}+\frac{\left(a_{1}+d-a_{0}\right)}{d} \mathcal{P}_{2}+\cdots+\frac{\left(a_{d-1}+d-a_{0}\right)}{d} \mathcal{P}_{d}\right)$,

where $\mathcal{P}_{1}=\sum_{k=1}^{d-1} P_{k 0}, \mathcal{P}_{2}=\sum_{k=0}^{d-1} P_{k 1}$ and $\mathcal{P}_{d}=\sum_{k=0}^{d-1} P_{k, d-1}$. We can find the extreme value of $\left(\mathcal{P}_{1}, \mathcal{P}_{2}, \cdots, \mathcal{P}_{d}\right)$ which is obtained under the product states $|\gamma\rangle=|\alpha\rangle_{1}|\alpha\rangle_{2}$ as

$$
\begin{aligned}
& \left\{\begin{array}{c}
\mathcal{P}_{1}=\left|\alpha_{0}\right|^{2}\left|\beta_{0}\right|^{2}+\left|\alpha_{1}\right|^{2}\left|\beta_{1}\right|^{2}+\cdots+\left|\alpha_{d-1}\right|^{2}\left|\beta_{d-1}\right|^{2} \\
-\frac{1}{d}|| \alpha_{0}|| \beta_{0}|+| \alpha_{1}|| \beta_{1}\left|e^{i \phi_{1}}+\cdots+\right| \alpha_{d-1}|| \beta_{d-1}\left|e^{i \phi_{d-1}}\right|^{2} \\
\mathcal{P}_{2}=\left|\alpha_{0}\right|^{2}\left|\beta_{1}\right|^{2}+\left|\alpha_{1}\right|^{2}\left|\beta_{2}\right|^{2}+\cdots+\left|\alpha_{d-1}\right|^{2}\left|\beta_{0}\right|^{2} \\
\vdots \\
\mathcal{P}_{d}=\left|\alpha_{0}\right|^{2}\left|\beta_{d-1}\right|^{2}+\left|\alpha_{1}\right|^{2}\left|\beta_{0}\right|^{2}+\cdots+\left|\alpha_{d-1}\right|^{2}\left|\beta_{d-2}\right|^{2}
\end{array}\right. \\
& \text { where }|\alpha\rangle_{1}=\left(\begin{array}{c}
\alpha_{0} \\
\alpha_{1} \\
\vdots \\
\alpha_{d-1}
\end{array}\right) \text { and }|\alpha\rangle_{2}=\left(\begin{array}{c}
\beta_{0} \\
\beta_{1} \\
\vdots \\
\beta_{d-1}
\end{array}\right) \text {. One can obtain the extreme points of the }
\end{aligned}
$$


$\left(\mathcal{P}_{1}, \mathcal{P}_{2}, \cdots, \mathcal{P}_{d}\right)$ as

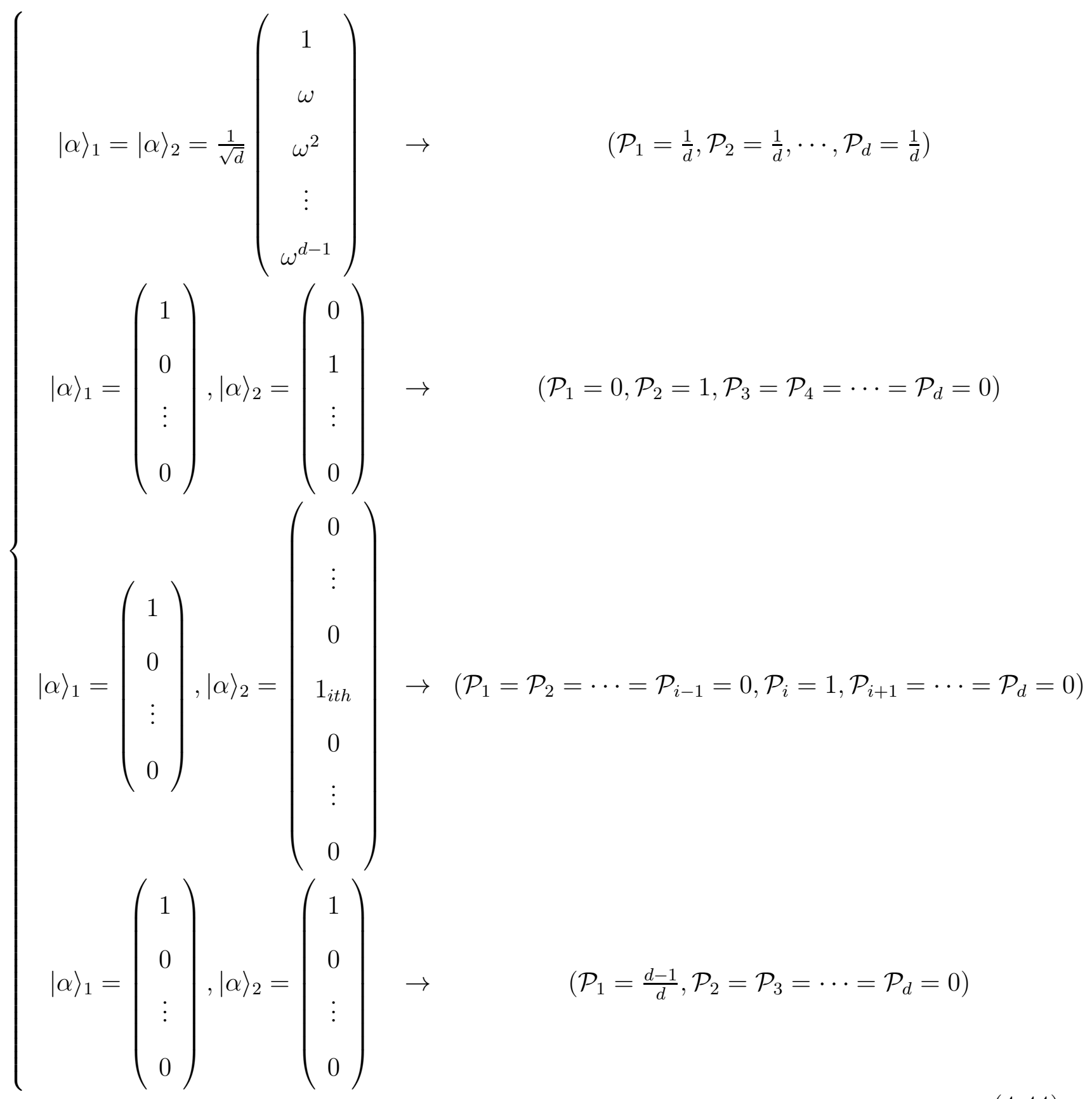

where $\omega=e^{\frac{2 \pi i}{d}}$.

The convex combination of all extreme points provide a convex or a feasible region, then 
we have the following optimization problem

$$
\left\{\begin{array} { c } 
{ \text { minimize } ( C _ { \gamma } ) = \frac { 1 } { ( ( d ^ { 2 } - 1 ) + ( 1 - d ) a _ { 0 } + a _ { 1 } + \cdots + a _ { d - 1 } ) } ( \mathcal { P } _ { 1 } + \frac { ( a _ { 1 } + d - a _ { 0 } ) } { d } \mathcal { P } _ { 2 } + \cdots + \frac { ( a _ { d - 1 } + d - a _ { 0 } ) } { d } \mathcal { P } _ { d } ) } \\
{ 1 - \frac { d } { d - 1 } \mathcal { P } _ { 1 } - \frac { d - 2 } { d - 1 } \mathcal { P } _ { 2 } - \mathcal { P } _ { 3 } - \cdots - \mathcal { P } _ { d } \leq 0 } \\
{ \text { subject to } }
\end{array} \left\{\begin{array}{c}
1-\frac{d}{d-1} \mathcal{P}_{1}-\frac{d-2}{d-1} \mathcal{P}_{3}-\mathcal{P}_{2}+\cdots+\mathcal{P}_{d} \leq 0 \\
\vdots \\
1-\mathcal{P}_{1}-\mathcal{P}_{2}-\cdots-\mathcal{P}_{d} \geq 0 \\
\mathcal{P}_{1}, \mathcal{P}_{2}, \cdots, \mathcal{P}_{d} \geq 0
\end{array}\right.\right.
$$

Analytically, we have been able to show that we will have violation only from the hyperplanes

$$
(d-1)-d \mathcal{P}_{1}-(d-1) \mathcal{P}_{i}-(d-2) \sum_{j \neq i=2}^{d} \mathcal{P}_{j}=0, i=2, \ldots d
$$

Now let us assume that the maximum value of the violation from the planes is $\Delta<1$. Thus, the equation of the plane passing through the new extreme points, parallel to the above plane, is obtained. Next we derive the intersection of the following adjacent planes

$$
\left\{\begin{array}{c}
d \mathcal{P}_{1}+(d-2) \mathcal{P}_{2}+(d-1) \mathcal{P}_{3}+\cdots+(d-1) \mathcal{P}_{d}-(d-1+\Delta)=0 \\
\vdots \\
d \mathcal{P}_{1}+(d-1) \mathcal{P}_{2}+(d-1) \mathcal{P}_{3} \cdots+(d-2) \mathcal{P}_{d}-(d-1+\Delta)=0 \\
\mathcal{P}_{1}+\mathcal{P}_{2}+\cdots+\mathcal{P}_{d}-1=0 \\
\mathcal{P}_{i}=0 \quad, i=1, \ldots, d \\
\mathcal{P}_{1}=\frac{d-1}{d} \quad, i=2, \ldots, d \\
\mathcal{P}_{i}=1 \quad \\
\mathcal{P}_{1}+\mathcal{P}_{2}+\cdots+\mathcal{P}_{d}=\frac{d-1}{d}
\end{array}\right.
$$

where new extreme points are obtained from intersecting the above hyperplanes. Next we calculate $C_{\gamma}$ for all the newly obtained extreme points and compare them with one another. Some easy calculations give the minimum value of the parameter $C_{\gamma}$ which is independent 
from $\Delta$ :

$$
\left(C_{\gamma}\right)_{\min }=\frac{d-1}{d} \frac{1+\frac{a_{\min }-a_{0}}{d}}{\left(\left(d^{2}-1\right)+(1-d) a_{0}+a_{1}+\cdots+a_{d-1}\right)}, \quad a_{\min }=\min \left\{a_{0}, a_{1}, \ldots, a_{d-1}\right\}
$$

then the critical value of the parameter $\mathrm{r}$ is obtained as

$$
\mathbf{r}_{c}=\frac{-d^{2} C_{\gamma_{\min }}}{1-d^{2} C_{\gamma_{\min }}}
$$

For $a_{0}=a_{1}=\cdots=a_{d-1}=1$ the parameter $\mathrm{r}$ reduces to $r_{c}=-d$ corresponding to the well known reduction map

$$
W_{\text {red }}=\sum_{i=0}^{d-1} \rho_{i}-d\left|\psi_{00}\right\rangle\left\langle\psi_{00}\right|
$$

On the other hand, EW (4-40) must have positive trace under any product state $|\gamma\rangle\langle\gamma|$. Thus the introduced $\mathrm{r}$ in (4-39) must satisfy

$$
r \geq r_{c} \Rightarrow-\frac{d\left(d-a_{0}\right)}{\left(a_{0}+\cdots+a_{d-1}-1\right)} \geq \frac{-d^{2} C_{\gamma_{\min }}}{1-d^{2} C_{\gamma_{\min }}}
$$

where the inequality is satisfied for all value of $1 \leq a_{0} \leq d-1$.

\section{$5 \quad$ Generalized $d \otimes d$ Choi entanglement witness of second type}

Similar to (4-35) one can define the second generalized $d \otimes d$ Choi entanglement witness by second set of Bell diagonal product states $\rho_{m}^{\prime}$ as

$$
W_{C h o i}^{\prime}=\sum_{i=0}^{d-1} a_{i} \rho_{i}^{\prime}-d\left|\psi_{00}\right\rangle\left\langle\psi_{00}\right|
$$

Comparing with (4-38) entanglement witness $W_{\text {Choi }}^{\prime}$ reduced to

$$
W_{C h o i}^{\prime}=\frac{1}{d\left(a_{0}+\cdots+a_{d-1}-1\right)}\left(-\left(d-a_{0}\right) I_{d^{2}}+d \sum_{k=1}^{d-1}\left|\psi_{0 k}\right\rangle\left\langle\psi_{0 k}\right|+\sum_{k=1}^{d-1}\left(a_{i}+d-a_{0}\right) \rho_{k}^{\prime}\right) .
$$


Comparing with BDEW (3-29) we have

$$
\mathbf{r}=-\frac{d\left(d-a_{0}\right)}{\left(a_{0}+\cdots+a_{d-1}-1\right)}
$$

By using (3-30) for non-negativity of the observable $W_{c h o i}^{\prime}$ we find the distributions $P_{i j}$ as a function of $q_{i j}$. The minimum value of $C_{\gamma}^{\prime}$ is obtained from the boundary of the feasible region, i.e., we have

$\left(C_{\gamma}\right)=\frac{1}{\left(\left(d^{2}-1\right)+(1-d) a_{0}+a_{1}+\cdots+a_{d-1}\right)}\left(\mathcal{P}_{1}^{\prime}+\frac{\left(a_{1}+d-a_{0}\right)}{d} \mathcal{P}_{2}^{\prime}+\cdots+\frac{\left(a_{d-1}+d-a_{0}\right)}{d} \mathcal{P}_{d}^{\prime}\right)$,

where $\mathcal{P}_{1}^{\prime}=\sum_{k=1}^{d-1} P_{0 k}, \mathcal{P}_{2}^{\prime}=\sum_{k=0}^{d-1} P_{1 k}$ and $\mathcal{P}_{d}^{\prime}=\sum_{k=0}^{d-1} P_{d-1, k}$. We can find the extreme value of $\left(\mathcal{P}_{1}^{\prime}, \mathcal{P}_{2}^{\prime}, \cdots, \mathcal{P}_{d}^{\prime}\right)$ which is obtained under the product states $|\gamma\rangle=|\alpha\rangle|\beta\rangle$ as

$$
\left\{\begin{array}{c}
\mathcal{P}_{1}^{\prime}=\frac{1}{d}\left(\sum_{k, l=0}^{d-1} \alpha_{l} \beta_{l+k}\left(\sum_{i=0}^{d-1} \bar{\alpha}_{l+i} \bar{\beta}_{l+k+i}\right)-\frac{1}{d}\left|\sum_{i=0}^{d-1}\right| \alpha_{i}|| \beta_{i}\left|e^{i \phi_{i}}\right|^{2}, \phi_{0}=0\right. \\
\mathcal{P}_{2}^{\prime}=\frac{1}{d}\left(\sum_{k, l=0}^{d-1} \alpha_{l} \beta_{l+k}\left(\sum_{i=0}^{d-1} \omega^{i} \bar{\alpha}_{l+i} \bar{\beta}_{l+k+i}\right)\right. \\
\vdots \\
\mathcal{P}_{d}^{\prime}=\frac{1}{d}\left(\sum_{k, l=0}^{d-1} \alpha_{l} \beta_{l+k}\left(\sum_{i=0}^{d-1} \omega^{(d-1) i} \bar{\alpha}_{l+i} \bar{\beta}_{l+k+i}\right)\right.
\end{array},\right.
$$


where $|\alpha\rangle=\left(\begin{array}{c}\alpha_{0} \\ \alpha_{1} \\ \vdots \\ \alpha_{d-1}\end{array}\right)$ and $|\beta\rangle=\left(\begin{array}{c}\beta_{0} \\ \beta_{1} \\ \vdots \\ \beta_{d-1}\end{array}\right)$. One can obtain the extreme points of the $\left(\mathcal{P}_{1}^{\prime}, \mathcal{P}_{2}^{\prime}, \cdots, \mathcal{P}_{d}^{\prime}\right)$ as

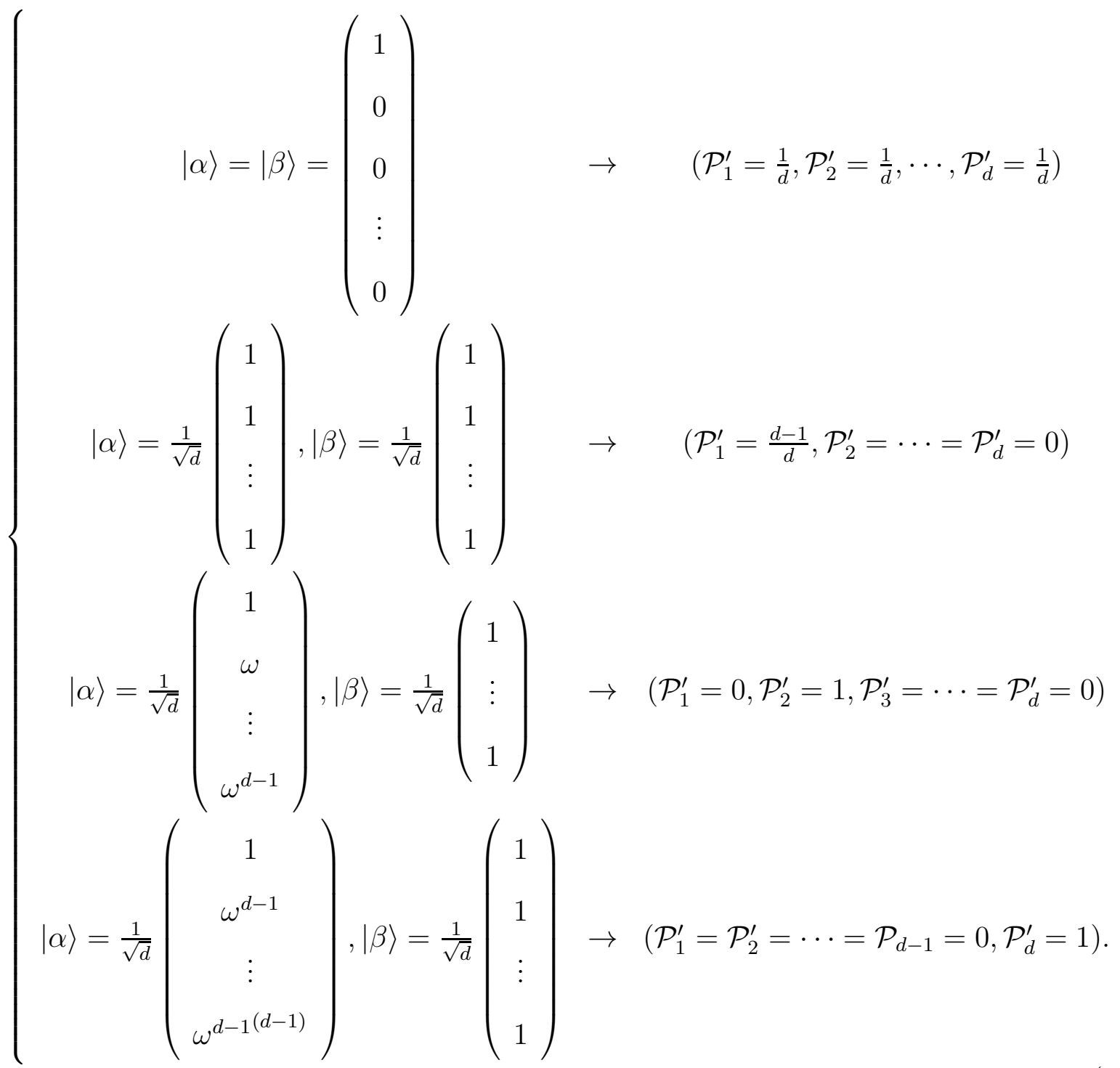

The convex combination of all extreme points provide a convex or a feasible region which is completely similar to feasible region of $W_{C h o i}$ entanglement witness (4-34) and then optimization problem is similar to previous optimization problem and finally $C_{\gamma_{\min }}$ is equal to (3-32) 
and critical value for $\mathrm{r}$ parameter is equal to (4-48).

\section{Non-decomposibility condition for generalized Choi entanglement witnesses}

As it is explained in first section, an entanglement witness $W$ is non-decomposable iff there exists a bound entangled state $\rho$ where $\operatorname{Tr}(\rho W)<0$. First we assume that Choi's EW is decomposable, hence it can be written as a convex sum of a positive operator $\mathrm{P}$ and partial transpose of a positive operator $\mathrm{Q}$ as follows

$$
W_{C h o i}=Q^{T_{A}}+P \text {. }
$$

To do so consider (4-49) reduction EW ( $\left.W_{\text {red }}\right)$ which is a optimal decomposable EW (For more

details see $[6,11])$ hence it can be written as partial transpose of positive operator $\tilde{Q}$ i.e., $W_{\text {red }}=\tilde{Q}^{T_{A}}$ where $\tilde{Q}=\sum_{i=0}^{d-1} \rho_{i}-\sum_{m, l=0}^{d-1} \omega^{m l}\left|\psi_{m l}\right\rangle\left|\psi_{m, d-l}\right\rangle$. Now using the reduction map one can decompose the Choi's EW of first type as

$$
W_{C h o i}=\lambda W_{\text {red }}+(1-\lambda) \tilde{P}
$$

where positive operator $\tilde{P}$ is

$$
\begin{gathered}
\tilde{P}=\left(\frac{a_{0}-d}{\left(\sum_{i=0}^{d-1} a_{i}-1\right) d}+\frac{\lambda}{d}\right)\left|\psi_{00}\right\rangle\left\langle\psi_{00}\left|+\left(\frac{a_{0}}{\left(\sum_{i=0}^{d-1} a_{i}-1\right) d}-\frac{\lambda}{d(d-1)}\right) \sum_{k=1}^{d-1}\right| \psi_{k 0}\right\rangle\left\langle\psi_{k 0}\right|+ \\
\sum_{i=1}^{d-1}\left(\frac{a_{i}}{\left(\sum_{i=0}^{d-1} a_{i}-1\right) d}-\frac{\lambda}{d(d-1)}\right) \rho_{i} \geq 0 .
\end{gathered}
$$

The above positive operators is Bell states diagonal, hence its eigenvalues, i.e., the coefficients of Bell states projection operators must be positive, therefore we should have

$$
\begin{gathered}
\lambda \geq \frac{d-a_{0}}{\left(\sum_{i=0}^{d-1} a_{i}-1\right)} \\
\lambda \leq \frac{a_{j}(d-1)}{\left(\sum_{i=0}^{d-1} a_{i}-1\right)}, j=0, \ldots, d-1 .
\end{gathered}
$$


Now, combining the inequalities (6-60) and (6-61) yields

$$
a_{i} \geq \frac{d-a_{0}}{d-1}, i=1, \ldots, d-1
$$

Also, by raining both sides of inequality (6-60) to (d-1) power and multiplying both sides of inequality (6-61), we get following inequality

$$
\frac{\left(d-a_{0}\right)^{d-1}}{\left(\sum_{i=0}^{d-1} a_{i}-1\right)^{d-1}} \leq \lambda^{d-1} \leq \frac{\left(a_{1} a_{2} \ldots a_{d-1}\right)^{d-1}}{\left(\sum_{i=0}^{d-1} a_{i}-1\right)^{d-1}} .
$$

The above inequality implies that the Choi's EW is decomposable as long as its parameters satisfy the following inequality

$$
a_{1} a_{2} \ldots a_{d-1} \geq \frac{\left(d-a_{0}\right)^{d-1}}{(d-1)^{d-1}} .
$$

For particular case of $3 \otimes 3$ Choi EW, $W_{C h o i}=a \rho_{0}+b \rho_{1}+c \rho_{2}-3\left|\psi_{00}\right\rangle\left\langle\psi_{00}\right|$ above inequality reduces to

$$
b c \geq \frac{(3-a)^{2}}{4}
$$

which is in agreement with [9].

If one of the inequalities (6-60) and (6-61) violates, then operator $\tilde{P}$ will not be positive operator any more, consequently one cannot say that whether Choi EW is decomposable or non-decomposable, because decomposition (6-58) is not unique. In these cases, in order to find non-decomposability conditions for $d \otimes d$ Choi's EW (4-35), we try to detect $d \otimes d$ PPT density matrix (2-21) by them, namely we should have

$$
\operatorname{Tr}\left[W_{C h o i} \rho_{P P T}\right]=(1-p)\left(\sum_{i=1}^{d-1} a_{i} \mu_{i}\right)+p\left(a_{0}-d\right)<0,
$$

which yields the following lower bounds for parameter $\mathrm{p}$

$$
p>\frac{\sum_{i=1}^{d-1} a_{i} \mu_{i}}{d+\sum_{i=1}^{d-1} a_{i} \mu_{i}-a_{0}} .
$$

Now, combining this lower bound with the corresponding upper one, due to PPT property of $d \otimes d$ density matrix (2-21), namely $p<\frac{1}{d}$, we get the following rang for parameter $\mathrm{p}$

$$
\frac{\sum_{i=1}^{d-1} a_{i} \mu_{i}}{d+\sum_{i=1}^{d-1} a_{i} \mu_{i}-a_{0}}<p<\frac{1}{d}
$$


therefore, non-decomposability condition is

$$
(d-1) \sum_{i=1}^{d-1} a_{i} \mu_{i}<d-a_{0}
$$

First we consider $3 \otimes 3$ systems Choi EW where non-decomposability condition (6-69) reduces to

$$
b \mu_{1}+c \mu_{2}<\frac{3-a}{2}
$$

Now choosing $\mu_{1}=1, \mu_{2}=0$ we get

$$
b<\frac{3-a}{2}
$$

on the other hand, the EW (4-36) condition implies that $c \geq \frac{3-a}{2}$. Similarly by choosing $\mu_{1}=0, \mu_{2}=1$ together with (4-36) we get

$$
b \geq \frac{3-a}{2}, c<\frac{3-a}{2}
$$

Summarizing above results, we can deduces that Choi's witness is decomposable if its parameters satisfy (6-64) and it is non-decomposable otherwise. Since violation of the inequalities (6-60) and (6-61) will be equivalent to non-decomposability conditions provided that it remains an EW. Since these conditions can be the same as the non-decomposability (6-69) simply by appropriate choice of $\mu_{i}, i=1,2$.

Again one can conclude that $d \otimes d$ entanglement witness is decomposable if its parameters satisfy (6-64), other wise it is non-decomposable. Discussion about the decomposability or non-decomposability conditions of $d \otimes d$ Choi's EW is similar to $3 \otimes 3$ case. Obviously if the parameters $a_{i}, i=1, \ldots, d-1$ satisfy condition (6-64) it is decomposable but if some of the inequalities (6-60) and (6-61) violate, then one can show that, they are equivalent to non-decomposability condition.

Decomposability condition of Choi's EW second type is similar to first type since, one write

$$
W_{\text {Choi }}^{\prime}-\lambda W_{\text {red }}=\left(\frac{a_{0}-d}{\left(\sum_{i=0}^{d-1} a_{i}-1\right) d}+\frac{\lambda}{d}\right)\left|\psi_{00}\right\rangle\left\langle\psi_{00}\left|+\left(\frac{a_{0}}{\left(\sum_{i=0}^{d-1} a_{i}-1\right) d}-\frac{\lambda}{d(d-1)}\right) \sum_{k=1}^{d-1}\right| \psi_{0 k}\right\rangle\left\langle\psi_{0 k}\right|
$$




$$
+\sum_{i=1}^{d-1}\left(\frac{a_{i}}{\left(\sum_{i=0}^{d-1} a_{i}-1\right) d}-\frac{\lambda}{d(d-1)}\right) \rho_{i}^{\prime} \geq 0,
$$

where the positivity of above operator yields results similar to (6-64).

Discussion about its non-decomposability is hard in general. Since it is hard to find a PPT state consisting of separable state. So we restrict ourselves in the remaining part of this section only to $3 \otimes 3 \mathrm{EW}$ second type. Now evaluating $\operatorname{Tr}\left[W_{C h o i} \rho_{P P T}^{\prime}\right]$ with $\rho_{P P T}^{\prime}$ given in (2-24), we get

$$
\operatorname{Tr}\left[W_{C h o i} \rho_{P P T}^{\prime}\right]<0 \Rightarrow \frac{\sum_{i=1}^{2} a_{i} \mu_{i}}{3+\sum_{i=1}^{2} a_{i} \mu_{i}-a_{0}}<p<\frac{1}{3},
$$

we see that non-decomposability condition is similar to (6-69), therefore decomposability and non-decomposability conditions of EW of second type is the same as with the first one.

\section{Conclusion}

It is shown that finding generalized $d \otimes d$ Choi Bell states diagonal entanglement witnesses can be reduced to an LP problem. A large group of non decomposable Choi entanglement witnesses have been defined by using Bell diagonal product states and corresponding $d \otimes d$ PPT states. We hope that in this way, one can study the Optimality and non-decomposibility of Choi entanglement witnesss for generic bipartite $d_{1} \otimes d_{2}$ systems and multipartite $d_{1} \otimes d_{2} \otimes \ldots \otimes d_{n}$, which are under investigation.

\section{References}

[1] A. Einstein, B. Podolsky, and N. Rosen, Phys. Rev. 47, 777 (1935).

[2] M. N. Nielsen and I. L. Chuang, Quantum computation and quantum information (Cambridge University Press, Cambridge, 2000).

[3] R. F. Werner, Phys. Rev. A 40, 4277 (1989). 
[4] B. M. Terhal, Phys. Lett. A271, 319 (2000).

[5] A. Jamiolkowski, Rep. Mat, Phys, 3, 275 (1972).

[6] M. A. Jafarizadeh, M. Rezaee, S. K. S. yagoobi Phys . Rev. 72, 062106 (2005).

[7] S. L. Woronowicz, Rep. on Math. Phys. 10, 165 (1976).

[8] M. Lewenstein, Quantum Information Theory, Institute for Theoretical Physics, Unversity of Hannover, March 31, (2004).

[9] M. D. Choi, Linear Algebra and its Applications 12, 95 (1975).

[10] S. Boyd and L. Vandenberghe, Convex Optimization, Cambridge University Press, (2004).

[11] R. Horodecki and M. Horodecki, Phys. Rev. A 54, 1838 (1996). 